\title{
Variation in use of targeted therapies for metastatic renal cell carcinoma: Results from a Dutch population-based registry
}

\author{
S. De Groot ${ }^{*}$, S. Sleijfer², W. K. Redekop ${ }^{1}$, E. Oosterwijk ${ }^{3}$, J. B. A. G. Haanen ${ }^{4}$, L. A. L. M. Kiemeney ${ }^{3,5}$
} and C. A. Uyl-de Groot ${ }^{1}$

\begin{abstract}
Background: For patients with metastatic renal cell carcinoma (mRCC), targeted therapies have entered the market since 2006. The aims of this study were to evaluate the uptake and use of targeted therapies for mRCC in The Netherlands, examine factors associated with the prescription of targeted therapies in daily clinical practice and study their effectiveness in terms of overall survival (OS).

Methods: Two cohorts from PERCEPTION, a population-based registry of mRCC patients, were used: a 2008-2010 Cohort $(n=645)$ and a 2011-2013 Cohort $(n=233)$. Chi-squared tests for trend were used to study time trends in the use of targeted therapy. Patients were grouped based on the eligibility criteria of the SUTENT trial, the trial that led to sunitinib becoming standard of care, to investigate the use of targeted therapies amongst patients fulfilling those criteria. Multi-level logistic regression was used to identify patient subgroups that are less likely to receive targeted therapies.

Results: Approximately one-third of patients fulfilling SUTENT trial eligibility criteria did not receive any targeted therapy (29\% in the 2008-2010 Cohort; $35 \%$ in the 2011-2013 Cohort). Patients aged 65+ years were less likely to receive targeted therapy in both cohorts and different risk groups (odds ratios range between 0.84-0.92); other factors like number of metastatic sites were of influence in some subgroups. Amongst treated patients, there was a decreasing trend in sunitinib use over time $(p=0.0061)$, and an increasing trend in pazopanib use $(p=0.0005)$.

Conclusions: Targeted therapies have largely replaced interferon-alfa as first-line standard of care. Nevertheless, many eligible patients in Dutch daily practice did not receive targeted therapies despite their ability to improve survival. Reasons for their apparent underutilisation should be examined more carefully.
\end{abstract}

Keywords: Metastatic renal cell carcinoma, Targeted therapy, Uptake and use, Overall survival, Population-based registry

\section{Background}

Kidney cancer accounts for about $3 \%$ of all cancers with an estimated incidence of 115,200 in Europe in 2012 [1]. Renal cell carcinoma (RCC) represents $90 \%$ of all kidney cancers [2]. The prognosis is relatively good for patients with localised disease, which can be treated with surgery, but the prognosis of patients with advanced or metastatic disease (mRCC) is poor [3].

\footnotetext{
* Correspondence: degroot@imta.eur.nl

${ }^{1}$ Institute of Health Policy and Management, Erasmus University Rotterdam, P.O. Box 1738, 3000 DR Rotterdam, The Netherlands

Full list of author information is available at the end of the article
}

Targeted therapies for $\mathrm{mRCC}$ have entered the market since 2006, sunitinib being the first. Sunitinib increased median progression-free survival (PFS) from five to 11 months [4], and overall survival (OS) from 22 to 26 months compared to interferon-alfa (IFN-a) in mRCC patients with a clear-cell histology [5]. Subsequently, it became standard of care for patients with a good or intermediate prognosis according to the Memorial Sloan Kettering Cancer Center (MSKCC) risk score [6]. Recently, the effectiveness of sunitinib was demonstrated in a broader 'real-world' population [7]. Bevacizumab (in combination with IFN-a) and pazopanib were added to 
guidelines as first-line therapies for patients with a good or intermediate prognosis in 2009 and 2010, respectively $[6,8]$. For patients with a poor prognosis, temsirolimus was recommended [6] following the results of a multicentre, phase III trial in mRCC patients without any restrictions in histologic type, showing an increase in OS from seven to 11 months compared to IFN-a [9]. Furthermore, a number of second-line therapies have been added to guidelines, such as sorafenib, everolimus and axitinib $[6,10]$.

Obviously, full and swift implementation of guidelines into clinical practice is essential to maximise the benefits of new therapies. However, the adoption of innovations in cancer care is generally quite heterogeneous, and differs between countries, and regions within countries [11]. A study by Jonsson et al. showed widespread use of sunitinib in the eight of the countries they studied, despite small differences between countries [12]. Sorafenib was widely prescribed in France, while a very low uptake and use in the United Kingdom and the United States were found. Besides between-country variation, Jonsson et al. found within-country variation in Sweden and suggested that more detailed information is needed on the use of first- and second-line therapies, to determine the extent of potential under- and overconsumption in different regions and different patient populations [12].

The aims of this study were to evaluate the uptake and use of targeted therapies for mRCC in The Netherlands, examine factors associated with the prescription of targeted therapies in daily clinical practice and study their effectiveness in terms of OS.

\section{Methods}

\section{Study population}

A population-based registry (entitled PERCEPTION) was created to include patients with mRCC. The PERCEPTION registry consisted of two parts; a retrospective study and a prospective study. In the retrospective study, eligible patients were selected from the Netherlands Cancer Registry (NCR), which maintains a cancer registration database of all cancer patients in The Netherlands. Inclusion criteria for the retrospective study comprised a diagnosis of mRCC (i.e. metastases at initial presentation) of any histological subtype. Patients diagnosed from January 2008 until December 2010 in 42 of 51 hospitals (both general and academic) in four regions, covering approximately half of the country, were included. All patients were followed for a minimum of three years or until death (2008-2010 Cohort).

The prospective study was designed differently in order to measure additional aspects of the disease, such as health-related quality of life (not reported in this study). In the prospective study, patients with RCC (all stages) of any histological subtype diagnosed from 2011 until June $30^{\text {th }} 2013$ in 25 of 32 hospitals (both general and academic) in three regions were included. In contrast to the 2008-2010 Cohort, this cohort also comprised patients with $\mathrm{mRCC}$ who were initially diagnosed with localised disease. Besides the NCR, the hospitals' financing systems were used to select eligible patients at an early phase (for quality of life measurements). All patients were followed until the end of 2013 or until death (2011-2013 Cohort).

\section{Data collection}

Data on baseline demographics, clinical and laboratory factors were retrospectively collected from individual patient records by using uniform case report forms to ensure consistent data collection. Furthermore, data on treatment schemes and treatment endpoints (e.g. survival) were collected. Laboratory factors, such as haemoglobin and corrected calcium levels, were standardised according to routinely used reference values. Data were collected by personnel of the NCR and data collection stopped at the end of 2013.

\section{Statistical analyses}

To study differences in the proportion of patients receiving targeted therapy per half a year chi-squared tests were used. Exact tests were used to study possible time trends in the use of different therapies amongst treated patients. Additionally, chi-squared tests for trend were conducted.

Then, the use of targeted therapies within risk groups was studied. Risk groups were created using a slightly modified version of the MSKCC risk score [3]; a time from initial diagnosis to metastatic diagnosis of less than one year was used as a risk factor instead of a time from initial diagnosis to initiation of treatment of less than one year, since many patients in the study population did not receive any targeted therapy, thereby making it impossible to calculate the time to treatment. Additionally, the WHO performance status was used instead of Karnofsky performance status.

Furthermore, patients were grouped based on the eligibility criteria of the SUTENT trial [4], the trial that led to sunitinib becoming standard of care, to investigate the use of targeted therapies amongst patients fulfilling those criteria. Patients who had a clear-cell subtype, a WHO performance status of 0 or 1 and no brain metastases were classified as fulfilling the SUTENT trial eligibility criteria.

To identify patient subgroups that are less likely to receive targeted therapies in daily clinical practice among patients fulfilling SUTENT trial eligibility criteria, multilevel mixed-effects logistic regression was used to account for between-hospital variance. At the patient-level, patient and disease characteristics were taken into 
account including baseline demographics, clinical and laboratory factors $[13,14]$. Backward selection was used to select the covariates for the models; any non-significant covariates were excluded from the models one at a time.

OS was calculated from the start of therapy until death from any cause or the date of last follow-up, whichever came first, using the Kaplan-Meier method. For patients not receiving any targeted therapy, OS was calculated from the date of diagnosis.

Missing data regarding baseline characteristics were handled using multiple imputations by chained equations. This method generated imputations based on a set of imputation models, one for each variable with missing values [15].

All analyses were performed separately for the 20082010 Cohort and the 2011-2013 Cohort, because of differences in inclusion criteria, patient selection and duration of follow-up. The significance level was set at $\alpha=$ 0.10. Data analyses were conducted using STATA statistical analysis software (StataCorp. 2013. Stata Statistical Software: Release 13. College Station, TX: StataCorp LP).

\section{Results}

Patient and disease characteristics of the 2008-2010 Cohort

714 patients newly diagnosed with mRCC between 2008 and 2010 were identified. Of these patients 69 were excluded (Additional file 1: Figure S1), leaving 645 patients for data analysis. These patients were uniformly distributed across the three-year period since 213 patients were diagnosed in 2008, 216 in 2009 and 216 in 2010. Median follow-up was 3.3 years (95 \% C.I.: 3.2-3.6).

Table 1 shows the patient and disease characteristics for this cohort. Median age was 66 years (range 23-93) and the majority of patients was male (66\%). The distribution of patients according to the MSKCC risk score showed a high proportion of patients (58 \%) with a poor prognosis (versus $42 \%$ with an intermediate prognosis). Since all patients in the 2008-2010 Cohort presented with metastatic disease, none of them had a favourable prognosis (i.e. time from initial diagnosis was less than one year). Additional file 1: Table S1 provides the observed patient and disease characteristics (without imputations).

\section{Uptake of targeted therapies and their use in daily clinical practice (2008-2010 Cohort)}

Table 2 shows the first-line therapies used in the 20082010 Cohort. 336/645 patients $(52 \%)$ received a firstline therapy with the majority $(282,84 \%)$ treated with sunitinib. The distribution of patients across first-line therapies (per half-year period) is presented in Fig. 1. There is evidence of a difference between the half-year periods in the proportion of patients receiving targeted therapy $(p=0.041)$, but the chi-squared test for trend did not yield a significant result. Furthermore, no shift was found in the use of first-line therapies amongst treated patients.

Of the 336 patients receiving first-line therapy, $101 \mathrm{pa}-$ tients $(30 \%)$ also received a second-line therapy, with everolimus being the most common (40\%), followed by sorafenib (28\%). There was an increasing trend in everolimus use over time $(p<0.0001)$ and a decreasing trend in sorafenib use $(p<0.0001)$; from 2010 onwards, everolimus largely replaced sorafenib.

\section{Use of targeted therapies amongst patients with an intermediate prognosis (2008-2010 Cohort)}

Forty-two percent $(269 / 645)$ of the patients in the 20082010 Cohort had an intermediate prognosis.

$105 / 269$ patients (39 \%) received no targeted therapy. Some $(n=15)$ of these patients received a metastasectomy (combined with a nephrectomy) with a possible curative intention, making systemic therapy redundant. 40 of the remaining 90 patients (44\%) who were given neither targeted therapy nor a metastasectomy (combined with a nephrectomy) fulfilled the SUTENT trial eligibility criteria, indicating that they might have been eligible for treatment with sunitinib or another targeted therapy. 164/269 patients (61 \%) received a first-line treatment; the majority was treated with sunitinib (145/ 164; $88 \%$ ). Of the 145 patients treated with sunitinib, 102 fulfilled the SUTENT trial eligibility criteria.

In patients fulfilling SUTENT trial eligibility criteria (including patients not receiving any targeted therapy and patients treated with sunitinib), patients with an abnormal neutrophil count (OR, $0.28 ; p=0.045)$ were less likely to receive sunitinib, whereas patients with more than one metastatic site (OR, $3.35 ; p=0.010)$ were more likely to receive sunitinib after adjustment for additional patient and disease characteristics (see frequencies in Table 3).

The median OS of eligible patients not receiving any targeted therapy was 18.6 months (95 \% C.I. 8.4-33.7). Table 4 presents the median OS in subgroups of patients with an intermediate prognosis treated with first-line sunitinib. Median OS of eligible patients treated with sunitinib was 14.8 months (95 \% C.I. 10.8-16.1). Note that a different starting point was used for the survival analysis (compared to the survival analysis in patients not receiving any targeted therapy). The mean time from diagnosis to start of first-line sunitinib was 4.3 months (standard deviation [SD] 6.0).

Median OS was 11.9 months (95 \% C.I. 6.5-18.3) for ineligible patients treated with sunitinib, which was not significantly shorter than the OS of eligible patients treated with sunitinib. No significant differences were observed within the other subgroups. 
Table 1 Patient and disease characteristics 2008-2010 Cohort and 2011-2013 Cohort

\begin{tabular}{|c|c|c|c|c|}
\hline \multirow{2}{*}{$\overline{\text { Sex - n (\%) }}$} & \multicolumn{2}{|c|}{ 2008-2010 Cohort: mRCC at the initial diagnosis $(n=621)$} & \multicolumn{2}{|c|}{ 2011-2013 Cohort: mRCC $(n=221)$} \\
\hline & & & & \\
\hline Female & 213 & $34 \%$ & 60 & $27 \%$ \\
\hline Male & 408 & $66 \%$ & 161 & $73 \%$ \\
\hline Median age - yr (range) & 66 & $23-93$ & 66 & $27-93$ \\
\hline \multicolumn{5}{|l|}{ Histology - n (\%) } \\
\hline Clear cell & 354 & $57 \%$ & 152 & $69 \%$ \\
\hline Other $^{a}$ & 267 & $43 \%$ & 69 & $31 \%$ \\
\hline \multicolumn{5}{|c|}{ WHO performance status - $\mathrm{n}(\%)$} \\
\hline $0-1$ & 430 & $69 \%$ & 178 & $81 \%$ \\
\hline $2-4$ & 191 & $31 \%$ & 42 & $19 \%$ \\
\hline \multicolumn{5}{|l|}{ Site of metastasis - $\mathrm{n}(\%)$} \\
\hline One & 206 & $33 \%$ & 87 & $39 \%$ \\
\hline more than one & 415 & $67 \%$ & 134 & $61 \%$ \\
\hline \multicolumn{5}{|l|}{ Liver metastasis - n (\%) } \\
\hline No & 509 & $82 \%$ & 175 & $79 \%$ \\
\hline Yes & 112 & $18 \%$ & 46 & $21 \%$ \\
\hline \multicolumn{5}{|l|}{ Lung metastasis - n (\%) } \\
\hline No & 173 & $28 \%$ & 74 & $33 \%$ \\
\hline Yes & 448 & $72 \%$ & 147 & $67 \%$ \\
\hline \multicolumn{5}{|l|}{ Bone metastasis - n (\%) } \\
\hline No & 393 & $63 \%$ & 158 & $71 \%$ \\
\hline Yes & 228 & $37 \%$ & 63 & $29 \%$ \\
\hline \multicolumn{5}{|l|}{ Brain metastasis - n (\%) } \\
\hline No & 571 & $92 \%$ & 200 & $90 \%$ \\
\hline Yes & 50 & $8 \%$ & 16 & $7 \%$ \\
\hline \multicolumn{5}{|l|}{ Haemoglobin - n (\%) } \\
\hline Normal & 205 & $33 \%$ & 85 & $38 \%$ \\
\hline$<L L N$ & 416 & $67 \%$ & 136 & $62 \%$ \\
\hline \multicolumn{5}{|l|}{ Neutrophil count - n (\%) } \\
\hline Normal & 383 & $62 \%$ & 152 & $69 \%$ \\
\hline$>$ ULN & 238 & $38 \%$ & 69 & $31 \%$ \\
\hline \multicolumn{5}{|l|}{ Platelet count - n (\%) } \\
\hline Normal & 452 & $73 \%$ & 159 & $72 \%$ \\
\hline$>$ ULN & 169 & $27 \%$ & 62 & $28 \%$ \\
\hline \multicolumn{5}{|l|}{ Albumin - n (\%) } \\
\hline Normal & 391 & $63 \%$ & 130 & $59 \%$ \\
\hline$<L L N$ & 230 & $37 \%$ & 91 & $41 \%$ \\
\hline \multicolumn{5}{|c|}{ Corrected serum calcium - n (\%) } \\
\hline Normal & 421 & $68 \%$ & 140 & $63 \%$ \\
\hline$>$ ULN & 200 & $32 \%$ & 81 & $37 \%$ \\
\hline \multicolumn{5}{|c|}{ Alkaline phosphatase - n (\%) } \\
\hline Normal & 432 & $70 \%$ & 152 & $69 \%$ \\
\hline$>$ ULN & 189 & $30 \%$ & 69 & $31 \%$ \\
\hline
\end{tabular}


Table 1 Patient and disease characteristics 2008-2010 Cohort and 2011-2013 Cohort (Continued)

\begin{tabular}{|c|c|c|c|c|}
\hline \multicolumn{5}{|c|}{ Lactate dehydrogenase - n (\%) } \\
\hline Normal & 372 & $60 \%$ & 179 & $81 \%$ \\
\hline$>1.5$ times ULN & 249 & $40 \%$ & 42 & $19 \%$ \\
\hline \multicolumn{5}{|c|}{ Comorbidities - n (\%) } \\
\hline $0-1$ & 356 & $57 \%$ & 151 & $68 \%$ \\
\hline$>1$ & 265 & $43 \%$ & 67 & $30 \%$ \\
\hline \multicolumn{5}{|c|}{ Time since RCC diagnosis } \\
\hline$>$ one year & NA & NA & 16 & $7 \%$ \\
\hline$<$ one year & NA & NA & 204 & $92 \%$ \\
\hline
\end{tabular}

NOTE: 24 patients in the 2008-2010 Cohort and 12 patients in the 2010-2013 Cohort were excluded from this table, since these patients received a metastasectomy (combined with a nephrectomy) with a possible curative intention, making systemic treatment redundant Abbreviations: LLN lower limit of normal, ULN upper limit of normal, NA not applicable

${ }^{a}$ mRCC was clinically established without histopathological confirmation in $17 \%$ of patients and mRCC was classified as not otherwise specified without further subtyping in $13 \%$ of patients (Cohort 2008-2010). It is likely that a substantial proportion of these patients had a clear cell subtype

Table 2 Treatment patterns 2008-2010 Cohort and 2011-2013 Cohort

\begin{tabular}{|c|c|c|c|c|c|c|c|c|c|c|c|c|}
\hline \multirow{3}{*}{$\begin{array}{l} \\
\text { No systemic } \\
\text { therapy }\end{array}$} & \multicolumn{6}{|c|}{ 2008-2010 Cohort: mRCC at the initial diagnosis } & \multicolumn{6}{|c|}{ 2011-2013 Cohort: mRCC } \\
\hline & \multicolumn{2}{|c|}{$\begin{array}{l}\text { All patients } \\
(n=645)\end{array}$} & \multicolumn{2}{|c|}{$\begin{array}{l}\text { Intermediate } \\
\text { prognosis } \\
(n=269)\end{array}$} & \multicolumn{2}{|c|}{$\begin{array}{l}\text { Poor prognosis } \\
(n=376)\end{array}$} & \multicolumn{2}{|c|}{$\begin{array}{l}\text { All patients } \\
(n=233)\end{array}$} & \multicolumn{2}{|c|}{$\begin{array}{l}\text { Favourable/ } \\
\text { intermediate } \\
\text { prognosis }(n=136)\end{array}$} & \multicolumn{2}{|c|}{$\begin{array}{l}\text { Poor prognosis } \\
(n=97)\end{array}$} \\
\hline & $\begin{array}{l}309 \\
(48 \%)\end{array}$ & & $\begin{array}{l}105 \\
(39 \%)\end{array}$ & & $\begin{array}{l}204 \\
(54 \%)\end{array}$ & & $\begin{array}{l}94 \\
(40 \%)\end{array}$ & & $\begin{array}{l}52 \\
(38 \%)\end{array}$ & & $\begin{array}{l}42 \\
(43 \%)\end{array}$ & \\
\hline First-line therapy & $\begin{array}{l}336 \\
(52 \%)\end{array}$ & $\begin{array}{l}336 \\
(100 \%)\end{array}$ & $\begin{array}{l}164 \\
(61 \%)\end{array}$ & $\begin{array}{l}164 \\
(100 \%)\end{array}$ & $\begin{array}{l}172 \\
(46 \%)\end{array}$ & $\begin{array}{l}172 \\
(100 \%)\end{array}$ & $\begin{array}{l}139 \\
(60 \%)\end{array}$ & $\begin{array}{l}139 \\
(100 \%)\end{array}$ & $\begin{array}{l}84 \\
(62 \%)\end{array}$ & $\begin{array}{l}84 \\
(100 \%)\end{array}$ & $\begin{array}{l}55 \\
(57 \%)\end{array}$ & $\begin{array}{l}55 \\
(100 \%)\end{array}$ \\
\hline Sunitinib & & $282(84 \%)$ & & $145(88 \%)$ & & $137(80 \%)$ & & $110(79 \%)$ & & $66(79 \%)$ & & $44(80 \%)$ \\
\hline Temsirolimus & & $24(7 \%)$ & & $5(3 \%)$ & & $19(11 \%)$ & & $3(2 \%)$ & & $1(1 \%)$ & & $2(4 \%)$ \\
\hline Sorafenib & & $11(3 \%)$ & & $7(4 \%)$ & & $4(2 \%)$ & & $4(3 \%)$ & & $3(4 \%)$ & & $1(2 \%)$ \\
\hline $\begin{array}{l}\text { Bevacizumab + } \\
\text { IFN-a }\end{array}$ & & $6(2 \%)$ & & $2(1 \%)$ & & $4(2 \%)$ & & $2(1 \%)$ & & $1(1 \%)$ & & $1(2 \%)$ \\
\hline Pazopanib & & $4(2 \%)$ & & $4(2 \%)$ & & $0(0 \%)$ & & $11(8 \%)$ & & $7(8 \%)$ & & $4(7 \%)$ \\
\hline IFN-a & & $3(1 \%)$ & & $0(0 \%)$ & & $3(2 \%)$ & & $1(1 \%)$ & & $0(0 \%)$ & & $1(2 \%)$ \\
\hline Everolimus & & $3(1 \%)$ & & $1(1 \%)$ & & $2(1 \%)$ & & $2(1 \%)$ & & $1(1 \%)$ & & $1(2 \%)$ \\
\hline $\begin{array}{l}\text { Pazopanib- } \\
\text { everolimus }\end{array}$ & & $0(0 \%)$ & & $0(0 \%)$ & & $0(0 \%)$ & & $3(2 \%)$ & & $3(4 \%)$ & & $0(0 \%)$ \\
\hline Other & & $3(1 \%)$ & & $0(0 \%)$ & & $3(2 \%)$ & & $3(2 \%)$ & & $2(2 \%)$ & & $1(2 \%)$ \\
\hline $\begin{array}{l}\text { Second-line } \\
\text { therapy }\end{array}$ & $\begin{array}{l}101 \\
(16 \%)\end{array}$ & $\begin{array}{l}101 \\
(100 \%)\end{array}$ & $\begin{array}{l}57 \\
(21 \%)\end{array}$ & $\begin{array}{l}57 \\
(100 \%)\end{array}$ & $\begin{array}{l}44 \\
(12 \%)\end{array}$ & $\begin{array}{l}44 \\
(100 \%)\end{array}$ & $\begin{array}{l}37 \\
(16 \%)\end{array}$ & $\begin{array}{l}37 \\
(100 \%)\end{array}$ & $\begin{array}{l}25 \\
(18 \%)\end{array}$ & $\begin{array}{l}25 \\
(100 \%)\end{array}$ & $\begin{array}{l}12 \\
(12 \%)\end{array}$ & $\begin{array}{l}12 \\
(100 \%)\end{array}$ \\
\hline Everolimus & & $40(40 \%)$ & & $26(46 \%)$ & & 14 (32 \%) & & $21(57 \%)$ & & $12(50 \%)$ & & $9(75 \%)$ \\
\hline Sorafenib & & $28(28 \%)$ & & $15(26 \%)$ & & $13(30 \%)$ & & 5 (14\%) & & $4(16 \%)$ & & $1(8 \%)$ \\
\hline Sunitinib & & 14 (14 \%) & & $8(14 \%)$ & & $6(14 \%)$ & & $1(3 \%)$ & & $1(4 \%)$ & & $0(0 \%)$ \\
\hline Temsirolimus & & 11 (11\%) & & $4(7 \%)$ & & 7 (16 \%) & & $3(8 \%)$ & & $2(8 \%)$ & & $1(8 \%)$ \\
\hline Pazopanib & & $4(4 \%)$ & & $2(4 \%)$ & & $2(5 \%)$ & & $5(14 \%)$ & & $4(16 \%)$ & & $1(8 \%)$ \\
\hline $\begin{array}{l}\text { Bevacizumab + } \\
\text { IFN-a }\end{array}$ & & $1(1 \%)$ & & $1(2 \%)$ & & $0(0 \%)$ & & $2(5 \%)$ & & $2(8 \%)$ & & $0(0 \%)$ \\
\hline Other & & $3(3 \%)$ & & $1(2 \%)$ & & $2(5 \%)$ & & $0(0 \%)$ & & $0(0 \%)$ & & $0(0 \%)$ \\
\hline
\end{tabular}




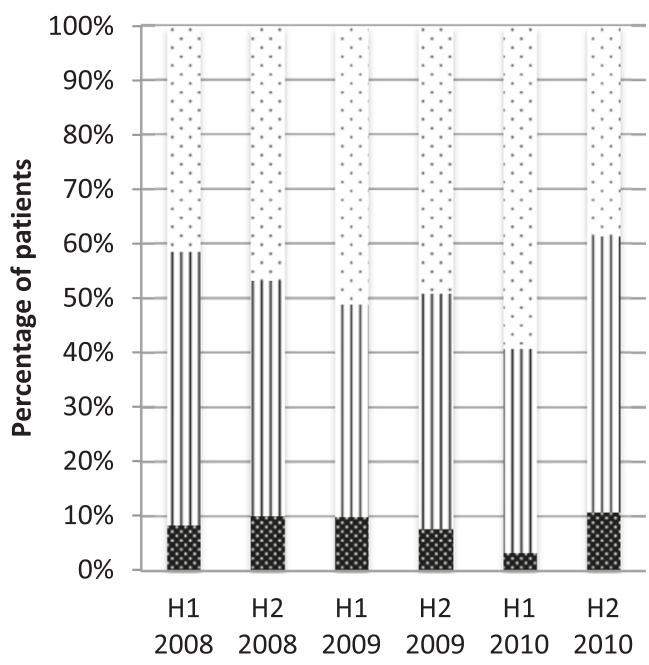

No systemic treatment

II First-line sunitinib

Other first-line therapy

Fig. 1 Use of first-line drugs over time per half a year (2008-2010 Cohort)

\section{Use of targeted therapies amongst patients with a poor} prognosis (2008-2010 Cohort)

Fifty-eight percent (376/645) of the patients in the 20082010 Cohort, had a poor prognosis. 204/376 patients (54\%) did not receive any targeted therapy. Of these patients, 9 patients received a metastasectomy (combined with a nephrectomy). 29 of the remaining 195 patients (15\%) who were given neither targeted therapy nor a metastasectomy (combined with a nephrectomy) fulfilled the SUTENT trial eligibility criteria. 172/376 (46 \%) patients received a first-line treatment, which was mainly sunitinib (137/376; $80 \%)$. Of the 137 patients treated with sunitinib, 70 fulfilled the SUTENT trial eligibility criteria.

Amongst patients fulfilling SUTENT trial eligibility criteria, older patients (OR, 0.90; $p=0.006)$ and patients with more than one comorbidity (OR, $0.26 ; p=0.090$ ) were less likely to receive sunitinib, whereas patients with more than one metastatic site (OR, 5.38; $p=0.034)$

Table 3 Patient subgroups that are more of less likely to receive targeted therapy while fulfilling SUTENT trial eligibility criteria

\begin{tabular}{|c|c|c|c|c|c|c|c|c|}
\hline & \multicolumn{4}{|c|}{ 2008-2010 Cohort: mRCC at the initial diagnosis } & \multicolumn{4}{|c|}{ 2011-2013 Cohort: mRCC } \\
\hline & \multicolumn{2}{|c|}{$\begin{array}{l}\text { Intermediate prognosis } \\
(n=142)\end{array}$} & \multicolumn{2}{|c|}{ Poor prognosis $(n=99)$} & \multicolumn{2}{|c|}{$\begin{array}{l}\text { Favourable/intermediate } \\
\text { prognosis }(n=70)\end{array}$} & \multicolumn{2}{|c|}{ Poor prognosis $(n=39)$} \\
\hline & $\begin{array}{l}\text { No targeted } \\
\text { therapy }(n=40)\end{array}$ & $\begin{array}{l}\text { Sunitinib } \\
(n=102)\end{array}$ & $\begin{array}{l}\text { No targeted } \\
\text { therapy }(n=29)\end{array}$ & $\begin{array}{l}\text { Sunitinib } \\
(n=70)\end{array}$ & $\begin{array}{l}\text { No targeted } \\
\text { therapy } n=25\end{array}$ & Sunitinib $n=45$ & $\begin{array}{l}\text { No targeted } \\
\text { therapy } n=13\end{array}$ & $\begin{array}{l}\text { Sunitinib } \\
n=26\end{array}$ \\
\hline \multicolumn{9}{|l|}{ Sex - n (\%) } \\
\hline Female & NS & NS & NS & NS & $2(8 \%)$ & $15(33 \%)$ & NS & NS \\
\hline Male & NS & NS & NS & NS & $23(92 \%)$ & $30(67 \%)$ & NS & NS \\
\hline Median age - yr (range) & & & $71(43-84)$ & $62(23-89)$ & $71(44-79)$ & $61(39-79)$ & $72(57-82)$ & $63(42-79)$ \\
\hline \multicolumn{9}{|l|}{ Site of metastasis - n (\%) } \\
\hline one & $25(62 \%)$ & 38 (37 \%) & $15(53 \%)$ & $20(29 \%)$ & NS & NS & NS & NS \\
\hline more than one & $15(38 \%)$ & $64(63 \%)$ & $14(47 \%)$ & $50(71 \%)$ & NS & NS & NS & NS \\
\hline \multicolumn{9}{|l|}{ Neutrophil count - n (\%) } \\
\hline normal & $27(68 \%)$ & $87(85 \%)$ & NS & NS & NS & NS & NS & NS \\
\hline$>$ ULN & $13(33 \%)$ & 15 (15 \%) & NS & NS & NS & NS & NS & NS \\
\hline \multicolumn{9}{|l|}{ Comorbidities } \\
\hline Zero or one & NS & NS & $15(52 \%)$ & $52(74 \%)$ & NS & NS & NS & NS \\
\hline More than one & NS & NS & $14(48 \%)$ & $18(26 \%)$ & NS & NS & NS & NS \\
\hline
\end{tabular}

NOTE: This table shows patient subgroups that are more or less likely to receive targeted therapy (i.e. first-line sunitinib) among patients fulfilling SUTENT trial eligibility criteria (according to the multi-level mixed-effects models). The multi-level models initially included all patient and disease characteristics as mentioned in Table 1 (besides hospital of diagnosis). Not significant (NS) means that this variable was not significantly associated to prescription of sunitinib at $a=0.10$ in a particular risk group/cohort Abbreviations: NS not significant 
Table 4 Overall survival in subgroups of patients treated with first-line sunitinib (Cohort 2008-2010 and Cohort 2011-2013)

\begin{tabular}{|c|c|c|c|c|c|c|c|}
\hline & \multicolumn{3}{|c|}{ 2008-2010 Cohort: mRCC at the initial diagnosis } & \multicolumn{3}{|c|}{ 2011-2013 Cohort: mRCC } \\
\hline & & $n$ & $\begin{array}{l}\text { Median OS in months } \\
(95 \% \text { C.I.) }\end{array}$ & p-value & $n$ & $\begin{array}{l}\text { Median OS in months } \\
(95 \% \text { C.I. })\end{array}$ & p-value \\
\hline \multicolumn{2}{|l|}{ All patients } & 282 & $9.1(7.2-11.1)$ & - & 109 & $10.1(7.2-13.8)$ & - \\
\hline \multirow[t]{2}{*}{ Fulfilling SUTENT trial eligibility criteria } & No & 110 & $6.5(4.9-8.9)$ & & 38 & $6.9(3.4-10.9)$ & \\
\hline & Yes & 172 & $11.9(8.8-14.6)$ & 0.0014 & 71 & 12.1 (8.9-NR) & 0.0074 \\
\hline \multirow[t]{2}{*}{ Brain metastases } & No & 261 & $9.3(7.6-11.9)$ & & 101 & $10.9(7.8-18.0)$ & \\
\hline & Yes & 21 & $4.3(2.1-11.5)$ & 0.0820 & 8 & $2.5(0.8-7.5)$ & 0.0125 \\
\hline \multirow[t]{2}{*}{ WHO performance status } & $0-1$ & 248 & $10.3(8.4-13.0)$ & & 100 & $11.3(7.8-18.0)$ & \\
\hline & $2-4$ & 34 & $3.3(1.8-6.2)$ & $<0.0001$ & 9 & $1.4(0.6-7.5)$ & $<0.0001$ \\
\hline \multirow[t]{2}{*}{ Histology } & Clear cell & 204 & $10.0(7.6-13.3)$ & & 81 & $10.6(7.2-20.3)$ & \\
\hline & Non-clear cell & 78 & $6.9(5.4-11.0)$ & 0.0809 & 28 & $10.0(3.5-13.8)$ & 0.3325 \\
\hline \multirow[t]{2}{*}{ Age } & $<65$ years & 162 & $8.9(6.5-10.8)$ & & 64 & $11.3(7.2-20.3)$ & \\
\hline & $>=65$ years & 120 & $10.0(6.5-13.8)$ & 0.8373 & 45 & $10.0(5.3-16.6)$ & 0.4294 \\
\hline \multicolumn{2}{|l|}{$\begin{array}{l}\text { Patients with an intermediate prognosis } \\
\text { (or favourable prognosis) }^{a}\end{array}$} & 145 & $14.6(11.5-16.0)$ & - & 65 & 16.6 (10.1-NR) & - \\
\hline \multirow[t]{2}{*}{ Fulfilling SUTENT trial eligibility criteria } & No & 43 & $11.9(6.5-18.3)$ & & 20 & 10.9 (2.7-NR) & \\
\hline & Yes & 102 & $14.8(10.8-16.1)$ & 0.2897 & 45 & 18.0 (10.1-NR) & 0.1212 \\
\hline \multirow[t]{2}{*}{ Brain metastases } & No & 136 & $14.6(10.7-16.0)$ & & 61 & 16.6 (10.9-NR) & \\
\hline & Yes & 9 & $11.9(4.3-29.3)$ & 0.8072 & 4 & 6.9 (2.5-NR) & 0.2282 \\
\hline \multirow[t]{2}{*}{ WHO performance status } & $0-1$ & 143 & $14.4(10.8-16.0)$ & & 64 & 16.6 (10.1-NR) & \\
\hline & $2-4$ & 2 & - & 0.2304 & 1 & - & 0.2471 \\
\hline \multirow[t]{2}{*}{ Histology } & Clear cell & 111 & $14.8(11.8-16.2)$ & & 49 & 18.0 (10.0-NR) & \\
\hline & Non-clear cell & 34 & $11.5(6.3-17.7)$ & 0.1954 & 16 & 13.8 (2.7-NR) & 0.3135 \\
\hline \multirow[t]{2}{*}{ Age } & $<65$ years & 87 & $10.8(7.2-15.7)$ & & 36 & 12.1 (7.2-NR) & \\
\hline & $>=65$ years & 58 & $16.1(12.4-18.8)$ & 0.2606 & 29 & 16.6 (8.5-NR) & 0.7157 \\
\hline \multicolumn{2}{|l|}{ Patients with a poor prognosis } & 137 & $6.1(4.9-7.7)$ & - & 44 & $6.5(3.4-10.0)$ & - \\
\hline \multirow[t]{2}{*}{ Fulfilling SUTENT trial eligibility criteria } & No & 67 & $4.7(3.3-6.9)$ & & 18 & $3.5(1.3-7.8)$ & \\
\hline & Yes & 70 & $6.8(5.3-10.7)$ & 0.0145 & 26 & 6.6 (3.8-NR) & 0.0720 \\
\hline \multirow[t]{2}{*}{ Brain metastases } & No & 125 & $6.5(5.3-8.4)$ & & 40 & $6.5(3.8-10.1)$ & \\
\hline & Yes & 12 & $2.1(0.7-4.2)$ & 0.0062 & 4 & 1.2 (0.8-NR) & 0.0134 \\
\hline \multirow[t]{2}{*}{ WHO performance status } & $0-1$ & 105 & $6.9(5.3-9.8)$ & & 36 & $6.6(3.8-10.1)$ & \\
\hline & $2-4$ & 32 & $3.1(1.4-5.5)$ & $<0.0001$ & 8 & $1.2(0.6-7.5)$ & 0.0087 \\
\hline \multirow[t]{2}{*}{ Histology } & Clear cell & 93 & $6.1(4.6-7.8)$ & & 32 & $6.5(2.7-10.1)$ & \\
\hline & Non-clear cell & 44 & $5.7(3.7-10.3)$ & 0.6585 & 12 & 4.1 (2.6-NR) & 0.9982 \\
\hline \multirow[t]{2}{*}{ Age } & $<65$ years & 75 & $6.9(4.9-9.8)$ & & 28 & $7.8(3.8-13.7)$ & \\
\hline & $>=65$ years & 62 & $5.4(3.8-6.8)$ & 0.4044 & 16 & $3.2(1.1-6.6)$ & 0.0256 \\
\hline
\end{tabular}

Abbreviations: C.I, confidence interval, $N R$ not reached

${ }^{a}$ Since all patients in the 2008-2010 Cohort presented with metastatic disease, none of the patients had a favourable prognosis (i.e. time from initial RCC diagnosis was less than one year)

were more likely to receive sunitinib (see frequencies in Table 3). Furthermore, a significant association was found between hospital of diagnosis and prescription of sunitinib $(p=0.0059)$.

Median OS of eligible patients not receiving any targeted therapy was 6.2 months (95\% C.I. 1.7-9.9). Table 4 shows the median OS in subgroups of patients with a poor prognosis treated with first-line sunitinib. Median OS of eligible patients treated with sunitinib was 6.8 months (95 \% C.I. 5.3-10.7). The mean time from diagnosis to start of first-line sunitinib was 2.9 months (SD 5.5).

Median OS was significantly reduced in poor-prognosis patients treated with sunitinib but not fulfilling the 
SUTENT trial eligibility criteria (4.7 months, 95 \% C.I. 3.3-6.9). Additionally, OS was significantly reduced in patients with brain metastases and patients with a WHO performance status of 2-4.

\section{Patient and disease characteristics of the 2011-2013 Cohort}

The second cohort study included 791 patients with (m)RCC diagnosed between 2011 and 2013. Of these patients, 233 had metastatic disease; 75 in 2011, 102 in 2012 and 55 in 2013 (one unknown). Median follow-up of the patients with mRCC was 1.2 years (95\% C.I. 1.1-1.4).

Table 1 shows the patient and disease characteristics of the patients with mRCC in this cohort. Median age was 66 years, and $73 \%(170 / 233)$ of the patients was men. Metastatic disease was present in $77 \%(179 / 233)$ of patients at the time of diagnosis, whereas $23 \%$ was initially diagnosed with localised disease. In this cohort, $4 \%$ of the patients with $\mathrm{mRCC}$ had a favourable prognosis, whereas $54 \%$ and $42 \%$ had an intermediate or poor prognosis, respectively.

\section{Uptake of targeted therapies and their use in daily clinical practice (2011-2013 Cohort)}

Table 2 shows the first-line therapies used in the 20112013 Cohort. During the follow-up period, 139/233 $(60 \%)$ patients received a first-line therapy; the majority $(110,79 \%)$ was treated with sunitinib. The distribution of patients across first-line therapies over time (half-year periods) is presented in Fig. 2. There were no significant differences between the half-year periods in the proportion of patients receiving targeted therapies. However, amongst treated patients, there was a decreasing trend in sunitinib use over time $(p=0.0061)$ and an increasing trend in pazopanib use $(p=0.0005)$.
Thirty-seven patients also received a second-line therapy within the follow-up period. The majority was treated with everolimus (57\%), but a decreasing trend in everolimus use over time was observed $(p=0.0020)$.

\section{Use of targeted therapies amongst patients with a} favourable or intermediate prognosis (2011-2013 Cohort) $136 / 233$ patients (58 \%) had a favourable or intermediate prognosis. 52/136 patients (38 \%) did not receive any targeted therapy within the follow-up period. However, 12 of these 52 patients received a metastasectomy (combined with a nephrectomy). 25 of the remaining $40 \mathrm{pa}$ tients $(63 \%)$ who were given neither targeted therapy nor a metastasectomy (combined with a nephrectomy) fulfilled the SUTENT trial eligibility criteria. In addition, 45 of the 66 patients treated with sunitinib fulfilled the SUTENT trial eligibility criteria.

Amongst patients fulfilling SUTENT trial eligibility criteria, males (OR, 0.12; $p=0.020$ ) and older patients (OR, $0.92 ; p=0.011$ ) were less likely to receive sunitinib after adjustment for additional patient and disease characteristics (see frequencies in Table 3).

Median OS of eligible patients not receiving any targeted therapy was 20.9 months (95 \% C.I. 7.4-not reached [NR]). Table 4 presents the median OS in subgroups of patients with a favourable or intermediate prognosis treated with first-line sunitinib. Median OS of eligible patients treated with sunitinib was 18.0 months (95\% C.I. 10.1-NR). The mean time from diagnosis to start of first-line sunitinib was 2.1 months (SD 3.3).

Median OS was 10.9 months (95 \% C.I. 2.7-NR) for patients treated with sunitinib but not fulfilling SUTENT trial eligibility criteria. No significant differences were observed within subgroups.

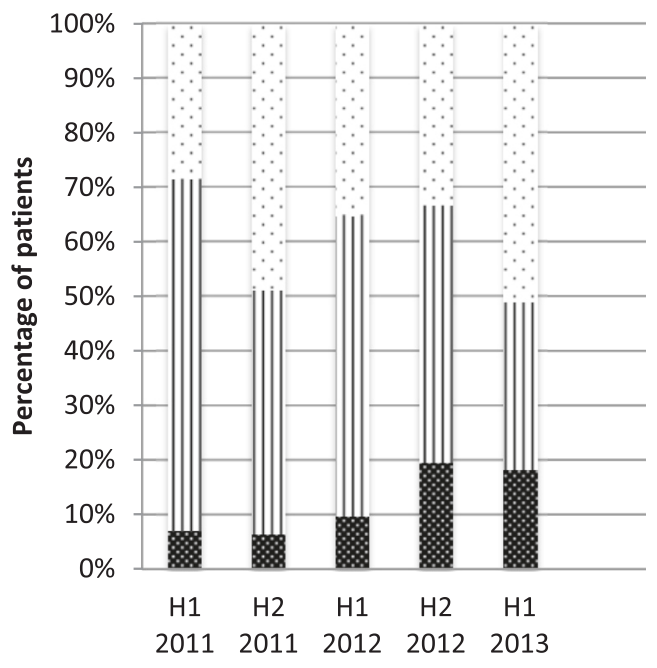

No systemic treatment

II First-line sunitinib

Other first-line therapy

Fig. 2 Use of first-line drugs over time per half a year (2011-2013 Cohort) 
Use of targeted therapies amongst patients with a poor prognosis (2011-2013 Cohort)

97/233 patients (42\%) had a poor prognosis. Forty-two patients $(43 \%)$ did not receive any targeted therapy; thirteen of these 42 patients (31 \%) fulfilled the SUTENT trial eligibility criteria. Of the 44 patients treated with sunitinib, 26 fulfilled the SUTENT trial eligibility criteria.

Of patients fulfilling SUTENT trial eligibility criteria, older patients (OR, $0.84 ; p=0.012)$ were less likely to receive sunitinib (see frequencies in Table 3). The unadjusted model showed a significant association between hospital of diagnosis and the prescription of sunitinib, but this association disappeared after adjustment for demographics, clinical and laboratory factors.

Median OS of eligible patients not receiving any targeted therapy was 3.4 months ( $95 \%$ C.I. 0.8 - NR). Table 4 shows the median OS in subgroups of patients with a poor prognosis treated with first-line sunitinib. Median OS of eligible patients treated with sunitinib was 6.6 months $(95 \%$ C.I. 3.8-NR). The mean time from diagnosis to start of first-line sunitinib was 1.9 months (SD 1.8).

Median OS was significantly reduced in patients not fulfilling the SUTENT trial eligibility criteria (3.5 months, 95 \% C.I. 1.3-7.8). Additionally, as in the 2008-2010 Cohort, median OS was significantly reduced in patients with brain metastases and patients with a WHO performance status of 2-4. OS was also significantly reduced in older patients.

\section{Discussion}

Since 2006, several new targeted therapies for mRCC have entered the market and randomised controlled trial (RCTs) have shown that these therapies improve survival $[4,5,9,16-27]$. This study examined the uptake and use of targeted therapies in The Netherlands. Not unexpected, targeted therapies, sunitinib in particular, have largely replaced IFN-a as first-line standard of care. Few patients were treated with bevacizumab (combined with IFN-a) or temsirolimus in the 2008-2013 period, even though these therapies were added to the ESMO guidelines in 2009 [6], and to Dutch guidelines in 2010 [6]. Pazopanib has only been recommended since 2010 [8], which partly explains why an increase in its use was only seen from 2012. Furthermore, there was a shift in the use of second-line therapies, where sorafenib was replaced by everolimus as the most frequent choice from 2010 onwards.

The median OS of patients with an intermediate prognosis treated with sunitinib in Dutch daily practice and fulfilling the SUTENT trial eligibility criteria was shorter than the median OS of patients in the SUTENT trial with an intermediate prognosis, i.e. 14.8 months (95\% C.I. 10.8-16.1) in the 2008-2010 Cohort compared to
20.7 months (95 \% C.I. 18.2-25.6) in the SUTENT trial [5]. However, the difference was much smaller for the 2011-2013 Cohort (median OS, 18.0 months (95 \% C.I. 10.1-NR)) compared to the SUTENT trial patients. Median OS of patients with a poor prognosis fulfilling the SUTENT trial eligibility criteria was similar to the median OS found in the SUTENT trial, i.e. 6.8 months (95 \% C.I. 5.3-10.7) in the 2008-2010 Cohort and 6.6 months (95\% C.I. 3.8-NR) in the 2011-2013 Cohort compared to 5.3 months (95 \% C.I. 4.2-10.0) in the SUTENT trial [5].

The median OS of patients with an intermediate prognosis treated with sunitinib in Dutch daily practice (regardless of their SUTENT trial eligibility status) was shorter than the OS in the expanded-access trial [7]. Median OS of patients with a poor prognosis was in line with the results of the expanded-access trial. The median OS of patients with an intermediate prognosis treated with sunitinib in Dutch daily practice was also shorter than the OS in a retrospective, non-interventional study in Australia [28]. These findings may indicate that the patients in the PERCEPTION registry with an intermediate risk had a worse prognosis than the patients with an intermediate risk in other studies.

While previous studies suggest that patients fulfilling SUTENT trial eligibility criteria have a survival benefit from first-line sunitinib [5], many eligible patients did not receive sunitinib (or any other targeted therapy) in daily practice. This was also seen in England where one in three patients with mRCC eligible for either sunitinib or pazopanib did not receive the drug [29]. Patients aged $65+$ years were less likely to receive targeted therapy than younger patients after adjustment for other factors. This age factor was found in patients with an intermediate prognosis (2011-2013 Cohort) and in patients with a poor prognosis (2008-2010 Cohort and 2011-2013 Cohort). There are several explanations for this association, including medical contraindications, other grounds for physician reluctance, and patient refusal. Additionally, patients with one metastatic site were less likely to receive sunitinib (according to the 2008-2010 Cohort results), which might be explained by patients with low volume but unresectable metastases whose targeted therapy is delayed. Nevertheless, most of these patients died within the follow-up period without receiving targeted therapy at any point in time. The reasons for apparent underutilisation of targeted therapies should be examined more carefully. While hospital-level factors may also affect utilisation and lead to between-hospital variation, we found no significant differences in the prescription of targeted therapy between hospitals, except for the patients with a poor prognosis in the 2008-2010 Cohort. However, the sample size per hospital was small and the statistical power to show a difference was therefore limited. 
Although this study mainly focussed on patients fulfilling SUTENT trial eligibility criteria, we found that many patients in daily clinical practice are different from patients included in RCTs. In the total study population, only $42 \%$ and $58 \%$ fulfilled the SUTENT trial eligibility criteria in the 2008-2010 Cohort and 2011-2013 Cohort, respectively. This was partly caused by the inclusion criteria of the PERCEPTION registry, which consisted of a diagnosis of $\mathrm{mRCC}$ (i.e. metastases at initial presentation in the 2008-2010 Cohort) of any histological subtype. Since many patients are excluded from clinical trials, such as patients with a non clear-cell subtype, patients with a WHO performance status of 2 to 4 and patients with brain metastases, one could argue that the results of these trials only apply to a subgroup of patients.

A limitation of this study is the amount of missing data in baseline characteristics, which is inherent to an observational study. To overcome this problem, multiple imputations by chained equations were conducted, which ensure that all patients are included in the analysis but simultaneously ensure that the uncertainties from missing data are retained [15]. Additionally, eligibility criteria, such as the presence of measurable disease and adequate organ function were not taken into account when determining whether patients fulfilled the SUTENT trial eligibility criteria, since data on these criteria were lacking in the PERCEPTION registry. As a consequence, some of the patients that we labelled as eligible in this study were not in fact eligible for targeted therapy. However, since we used WHO performance status to classify patients, and since we expect a relationship between WHO performance status and organ function, we believe that this could only have had a limited effect on our conclusions about the uptake and use of targeted therapies. Furthermore, the follow-up length of the 2011-2013 Cohort was limited. As a consequence, patients might have received targeted therapy after the follow-up period, leading to an underestimate of actual targeted therapy use. However, this limitation is only relevant for patients treated later in the 2011-2013 period who did not die. Lastly, OS was calculated from the date of diagnosis (i.e. metastatic disease) for patients not receiving any targeted therapy and from the start of therapy for patients treated with targeted therapy; as a consequence a comparison between the two is impossible. This approach was based on the one used in other studies to enable comparisons between the OS of patients treated with sunitinib in our study with the OS of patients treated with sunitinib in other studies $[5,7,28]$.

\section{Conclusions}

In conclusion, targeted therapies, sunitinib in particular, have largely replaced IFN-a as the first-line standard of care in The Netherlands. Nevertheless, many patients in
Dutch daily practice fulfilling SUTENT trial eligibility criteria did not receive sunitinib (or any other targeted therapy) even though it could improve their survival. For example, older patients were less likely to receive sunitinib, perhaps because physicians are reluctant to prescribe it. The reasons for apparent underutilisation of targeted therapies should be examined more carefully.

\section{Additional file}

Additional file 1: Figure S1. Patient enrolment. Table S1. Patient and disease characteristics (observed and imputed) 2008-2010 Cohort and 2011-2013 Cohort. (DOCX 57 kb)

\section{Abbreviations}

C.I., confidence interval; IFN-a, interferon-alfa; mRCC, metastatic renal cell carcinoma; MSKCC, Memorial Sloan Kettering Cancer Center; NCR, Netherlands Cancer Registry; NR, not reached; OS, overall survival; PERCEPTION, PharmacoEconomics in Renal CEll carcinoma: a PopulaTION-based registry; PFS, progression-free survival; RCC, renal cell carcinoma; RCT, randomised controlled trial; SD, standard deviation

\section{Acknowledgments}

The PERCEPTION registry was financially supported by the Netherlands Organisation for Health Research and Development (grant number: 152001014). Additional financial support was provided by Pfizer BV (formerly Wyeth Pharmaceuticals BV) and Roche Nederland BV. The authors would like to thank the registration team of the Netherlands Comprehensive Cancer Organisation for data collection.

\section{Funding}

The PERCEPTION registry was supported by the Netherlands Organisation for Health Research and Development [grant number 152001014]; Pfizer (formerly Wyeth Pharmaceuticals BV); and Roche Nederland BV. All funding bodies were involved in the design of the PERCEPTION registry, but not in the collection, analysis and interpretation of data. Pfizer provided feedback on a draft of the manuscript.

\section{Availability of data and materials}

The dataset supporting the conclusions of this article is available at request from the corresponding author.

\section{Author's contribution}

SG, SS, EO, JH, LK and CU have made substantial contributions to conception and design of the PERCEPION-registry. SG, SS, WR and CU have made substantial contributions to analysis and interpretation of data. All authors have been involved in drafting or revising the manuscript, and all authors have given final approval of the version to be published.

\section{Competing interests}

SS participates in a speakers bureau for GlaxoSmithKline. JH receives consultancy fees from Pfizer. LK received an unrestricted research grant from Pfizer to extend the data collection of the PERCEPTION registry. CU received unrestricted research grants from Pfizer (formerly Wyeth Pharmaceuticals BV) and Roche Nederland BV to support the PERCEPTION registry. All remaining authors declared no conflict of interest.

\section{Consent for publication}

Not applicable.

\section{Ethics approval and consent to participate}

The research protocol was approved by the medical ethics committee of Radboud university medical center in Nijmegen (CMO Region ArnhemNijmegen) in May 2010 (registration number: 2010/059). Informed consent for the current study was not required, because the data-collection was retrospective in design. 


\section{Author details}

Institute of Health Policy and Management, Erasmus University Rotterdam, P.O. Box 1738, 3000 DR Rotterdam, The Netherlands. ${ }^{2}$ Department of Medical Oncology and Cancer Genomics Netherlands, Erasmus MC Cancer Institute, Rotterdam, The Netherlands. ${ }^{3}$ Department of Urology, Radboud Institute for Molecular Life Sciences, Radboud University Medical Center, Nijmegen, The Netherlands. ${ }^{4}$ Department of Medical Oncology, The Netherlands Cancer Institute-Antoni van Leeuwenhoek Hospital, Amsterdam, The Netherlands. ${ }^{5}$ Department for Health Evidence, Radboud Institute for Health Sciences, Radboud University Medical Center, Nijmegen, The Netherlands.

\section{Received: 6 October 2015 Accepted: 2 June 2016}

Published online: 11 June 2016

\section{References}

1. Ferlay J, Steliarova-Foucher E, Lortet-Tieulent J, Rosso S, Coebergh JW, Comber $\mathrm{H}$, et al. Cancer incidence and mortality patterns in Europe: estimates for 40 countries in 2012. Eur J Cancer. 2013:49:1374-403.

2. Ljungberg B, Campbell SC, Choi HY, Jacamin D, Lee JE, Weikert S, et al. The epidemiology of renal cell carcinoma. Eur Urol. 2011;60:615-21.

3. Motzer RJ, Bacik J, Murphy BA, Russo P, Mazumdar M. Interferon-alfa as a comparative treatment for clinical trials of new therapies against advanced renal cell carcinoma. J Clin Oncol. 2002;20:289-96.

4. Motzer RJ, Hutson TE, Tomczak P, Michaelson MD, Bukowski RM, Rixe O, et al. Sunitinib versus interferon alfa in metastatic renal-cell carcinoma. $\mathrm{N}$ Engl J Med. 2007:356:115-24

5. Motzer RJ, Hutson TE, Tomczak P, Michaelson MD, Bukowski RM, Oudard S, et al. Overall survival and updated results for sunitinib compared with interferon alfa in patients with metastatic renal cell carcinoma. J Clin Oncol. 2009:27:3584-90

6. Escudier B, Kataja V, ESMO Guidelines Working Group. Renal cell carcinoma: ESMO clinical recommendations for diagnosis, treatment and follow-up. Ann Oncol. 2009;20 Suppl 4:81-2.

7. Gore ME, Szczylik C, Porta C, Bracarda S, Bjarnason GA, Oudard S, et al. Final results from the large sunitinib global expanded-access trial in metastatic renal cell carcinoma. Br J Cancer. 2015;113:12-9.

8. Escudier B, Kataja V, ESMO Guidelines Working Group. Renal cell carcinoma: ESMO Clinical Practice Guidelines for diagnosis, treatment and follow-up. Ann Oncol. 2010;21 Suppl 5:v137-9.

9. Hudes G, Carducci M, Tomczak P, Dutcher J, Figlin R, Kapoor A, et al. Temsirolimus, interferon alfa, or both for advanced renal-cell carcinoma. N Engl J Med. 2007:356:2271-81.

10. Escudier B, Eisen T, Porta C, Patard JJ, Khoo V, Algaba F, et al. Renal cell carcinoma: ESMO Clinical Practice Guidelines for diagnosis, treatment and follow-up. Ann Oncol. 2012;23 Suppl 7:vii65-71.

11. Uyl-de Groot CA, de Vries EGE, Verweij J, Sullivan R. Dispelling the myths around cancer care delivery: It's not all about costs. J Cancer Policy. 2014:2:22-9.

12. Jönsson B, Ramsey SD, Wilking N. Cost effectiveness in practice and its effect on clinical outcomes. J Cancer Policy. 2014;2:12-21.

13. Sun M, Shariat SF, Cheng C, Ficarra V, Murai M, Oudard S, et al. Prognostic factors and predictive models in renal cell carcinoma: a contemporary review. Eur Urol. 2011;60:644-61.

14. Motzer RJ, Escudier B, Bukowski R, Rini Bl, Hutson TE, Barrios CH, et al. Prognostic factors for survival in 1059 patients treated with sunitinib for metastatic renal cell carcinoma. Br J Cancer. 2013;108:2470-7.

15. White IR, Royston $P$, Wood AM. Multiple imputation using chained equations: Issues and guidance for practice. Stat Med. 2011;30:377-99.

16. Escudier B, Eisen T, Stadler WM, Szczylik C, Oudard S, Siebels M, et al. Sorafenib in advanced clear-cell renal-cell carcinoma. N Engl J Med. 2007;356:125-34

17. Escudier B, Eisen T, Stadler WM, Szczylik C, Oudard S, Staehler M, et al. Sorafenib for treatment of renal cell carcinoma: Final efficacy and safety results of the phase III treatment approaches in renal cancer global evaluation trial. J Clin Oncol. 2009;27:3312-8.

18. Escudier B, Pluzanska A, Koralewski P, Ravaud A, Bracarda S, Szczylik C, et al. Bevacizumab plus interferon alfa-2a for treatment of metastatic renal cell carcinoma: a randomised, double-blind phase III trial. Lancet. 2007;370:2103-11.

19. Escudier B, Bellmunt J, Negrier S, Bajetta E, Melichar B, Bracarda S, et al. Phase III trial of bevacizumab plus interferon alfa-2a in patients with metastatic renal cell carcinoma (AVOREN): final analysis of overall survival. J Clin Oncol. 2010:28:2144-50.

20. Rini BI, Halabi S, Rosenberg JE, Stadler WM, Vaena DA, Ou SS, et al. Bevacizumab plus interferon alfa compared with interferon alfa monotherapy in patients with metastatic renal cell carcinoma: CALGB 90206. J Clin Oncol. 2008:26:5422-8.

21. Rini BI, Halabi S, Rosenberg JE, Stadler WM, Vaena DA, Archer $L$, et al. Phase III trial of bevacizumab plus interferon alfa versus interferon alfa monotherapy in patients with metastatic renal cell carcinoma: final results of CALGB 90206. J Clin Oncol. 2010;28:2137-43.

22. Motzer RJ, Escudier B, Oudard S, Hutson TE, Porta C, Bracarda S, et al. Efficacy of everolimus in advanced renal cell carcinoma: a double-blind, randomised, placebo-controlled phase III trial. Lancet. 2008;372:449-56.

23. Motzer RJ, Escudier B, Oudard S, Hutson TE, Porta C, Bracarda S, et al. Phase 3 trial of everolimus for metastatic renal cell carcinoma : final results and analysis of prognostic factors. Cancer. 2010;116:4256-65.

24. Sternberg CN, Davis ID, Mardiak J, Szczylik C, Lee E, Wagstaff J, et al. Pazopanib in locally advanced or metastatic renal cell carcinoma: results of a randomized phase III trial. J Clin Oncol. 2010;28:1061-8.

25. Sternberg CN, Hawkins RE, Wagstaff J, Salman P, Mardiak J, Barrios CH, et al. A randomised, double-blind phase III study of pazopanib in patients with advanced and/or metastatic renal cell carcinoma: final overall survival results and safety update. Eur J Cancer. 2013;49:1287-96.

26. Rini Bl, Escudier B, Tomczak P, Kaprin A, Szczylik C, Hutson TE, et al. Comparative effectiveness of axitinib versus sorafenib in advanced renal cell carcinoma (AXIS): a randomised phase 3 trial. Lancet. 2011;378:1931-9.

27. Motzer RJ, Escudier B, Tomczak P, Hutson TE, Michaelson MD, Negrier S, et al. Axitinib versus sorafenib as second-line treatment for advanced renal cell carcinoma: overall survival analysis and updated results from a randomised phase 3 trial. Lancet Oncol. 2013;14:552-62.

28. Day D, Kanjanapan Y, Kwan E, Yip D, Lawrentschuk N, Andrews M, et al. Patterns of care for metastatic renal cell carcinoma in Australia. BJU Int. 2015:116 Suppl 3:36-41.

29. Prescribing and Primary Care team, Health and Social Care Information Centre: Use of NICE appraised medicines in the NHS in England - 2012 experimental statistics. Leeds, United Kingdom: Health and Social Care Information Centre; 2014.

\section{Submit your next manuscript to BioMed Central and we will help you at every step:}

- We accept pre-submission inquiries

- Our selector tool helps you to find the most relevant journal

- We provide round the clock customer support

- Convenient online submission

- Thorough peer review

- Inclusion in PubMed and all major indexing services

- Maximum visibility for your research

Submit your manuscript at www.biomedcentral.com/submit
) Biomed Central 